\title{
Cortical Evoked Potentials by Photic Stimulation at Various Adaptation Levels of Retina in Man
}

\author{
By \\ Mituru Ebe, Tomohisa Mikami and Hirotaka Ito \\ From Department of Applied Physiology, the Toranomon General \\ Hospital, Akasaka Aoi Cho, Minato Ku, Tokyo \\ (Received for publication, April 18, 1963)
}

\section{INTRODUCTION}

Monnier, ${ }^{1-3)}$ Cobb,, ${ }^{4,5)}$ Ciganék, ${ }^{6-8)}$ Balen and Henkes,,${ }^{9,10)}$ Laue, ${ }^{11.12)}$, Calvet, Contamin and Hirsch et $a l{ }^{13-15)}$ have studied on photically evoked potentials of the visual cortex in man, and thus at the present time physiological and anatomical correlation of the evoked potential to the visual cortex is being elucidated step by step by the method of evoked potentials and other physiological methods.

In our previous paper ${ }^{16)}$ the changes of the evoked potential depending upon the intensity of photic stimulation were reported. In the present paper the results of similar experiments carried out under light adaptation of various levels will be reported.

\section{METHOD}

37 subjects ranging from 11 to 70 years of age were used. The subjects were placed supine in a room with white walls illuminated by photoreflector lamps which were placed at a lower level than a bed height. The luminosity of adapting light was measured by a photometer at the position of the eye of the subject who was viewing the ceilling and was varied in 4 steps, that is, 0 (darkness), 4, 30, and 60 lux by varying the intensity of the light source.

Stimulation was provided from a Xenon lamp with a hyperbolic reflector which was fixed at a distance of $30 \mathrm{~cm}$ away from subject's eyes. The flash of light was white, within $5 \mu$ sec in duration and its maximum out-put energy was 7 ws per flash. The intensity of light was reduced to various degrees by filters of $\mathrm{X}$-ray films having transparency of $10 \%$.

The evoked potentials were led monopolarly or bipolarly from electrodes which were placed at the occipital protuberance and $3 \mathrm{~cm}$ below it, and recorded

江部 充, 三上智久，伊策弘多加 
by the superimposition method of Dawson. ${ }^{17)}$ The time interval of stimuli was over 1 sec, and the stimulus was given while the EEG was showing an activation pattern.

\section{RESULTS}

1. Changes of evoked potentials by the maximum intensity of the stimulus at various adaptation levels

In our experiments, the evoked potentials were observed at four adaptation levels of the retina, i.e. darkness, 4, 30 and 60 lux. As reported in the previous paper, ${ }^{18)}$ the evoked potentials led from the occipital protuberance in a dark room were classified into four groups and their average figure consisted of the initial positive small deflection with a latency of 20 to $30 \mathrm{msec}$, followed by negative waves with the culmination time of $44 \mathrm{msec}$, positive waves of $61 \mathrm{msec}$, negative waves of $83 \mathrm{msec}$, positive waves of $108 \mathrm{msec}$, negative waves of $136 \mathrm{msec}$ and positive waves of $169 \mathrm{msec}$ successively. These alternating deflections were numbered from 1 to 7 in order.

When the adaptation level was varied from darkness to light of various intensity, the form of the evoked potentials showed various changes. However, it seems that there are two main tendencies in these variable changes, that is, decrease in amplitude and changes of relative size of components.

As the intensity of adaptation light was increased, the decrease in amplitude of evoked potential was observed in 13 of 37 cases. In these cases, generally, every component of the evoked potential decreased, but the rate of decrease was different from component to component. Some examples are shown in Fig. 1. As illustrated in Fig. 1a, every component of the evoked potential decreased in amplitude gradually by changing the adaptation level successively from dark till light of 60 lux, especially in the stage of 4 lux the 5th positive deflection decreased remarkably in amplitude and in 60 lux the 4th negative wave was predominant. In Fig. 1b, as in other cases, the decrease in amplitude of the 3rd positive deflection was conspicuous in the adaptation to 30 lux and the 4th negative wave could still be observed at the adaptation level of 60 lux. In Fig. 1c, as in other cases, every component of evoked potential showed little change and the 4th negative component was predominant, but their amplitudes decreased at the adaptation level of 60 lux.

Moreover, in 7 cases out of 37 the form of evoked potential remained almost constant, and even at the adaptation level of 60 lux there occurred little decrease in amplitude. An example is shown in Fig. 2.

Another general tendency was as follows: The 4th negative wave which was a predominant component in the dark adaptation decreased in amplitude in the light adaptation and the 6th negative wave became larger than the 4th component. As shown in Fig. 3a, both the 4th and 6th negative waves were 


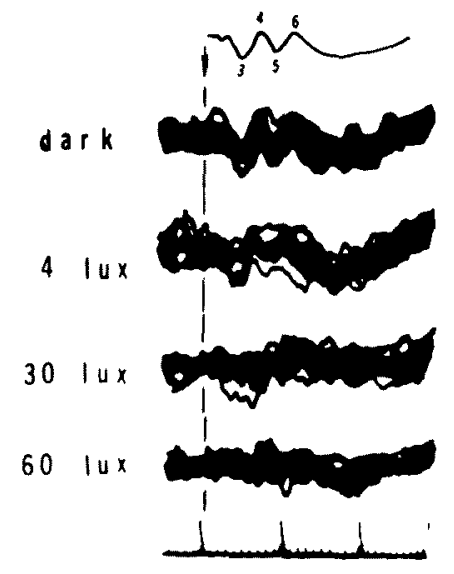

Fig. 1a.

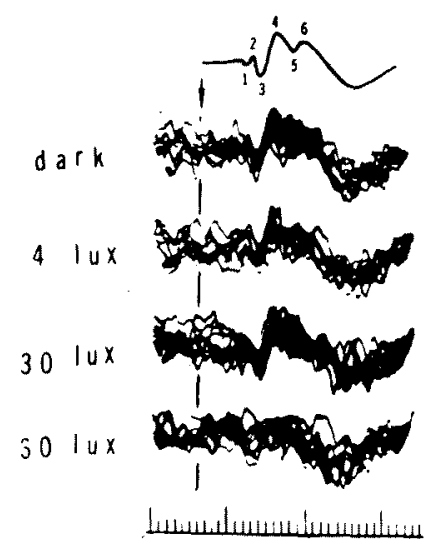

Fig. 1c.

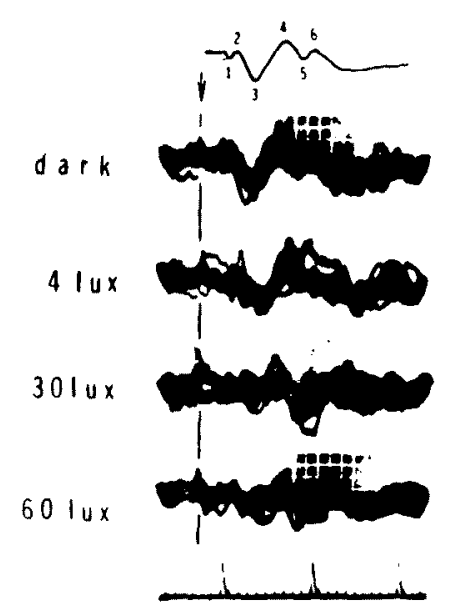

Fig. Ilb

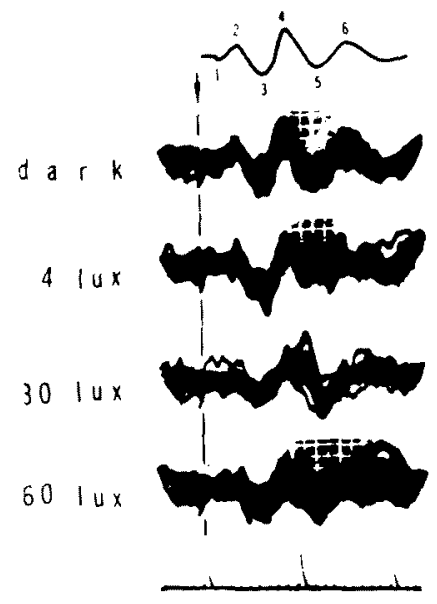

Fig. 2.

Fig. la, b and c. Photically evoked potentials at four adaptation levels of retina. The uppermost figure is a schema of the potential in dark. Vertical line; photic stimulation. Time; 10 and 100 msec.

Fig. 2. See text.

distinctly large in the dark, but in the light adaptation to 30 lux the 4 th wave became smaller and the 6 th wave remained as it was, although at the adaptation level of 60 lux both components became again almost equal in amplitude. In Fig. $3 \mathrm{~b}$ and $3 \mathrm{c}$, as in other cases, accompanying the shift of adaptation level from dark till 60 lux the 6 th wave became dominant over the 4 th wave in spite of the sustained amplitude of the 4 th wave. In 14 cases out of 37 such a change in 


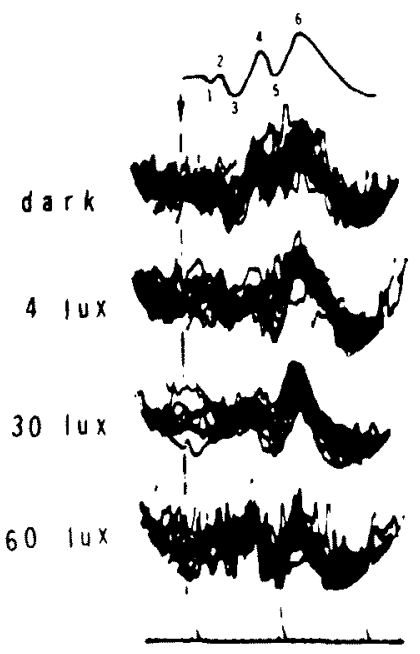

Fig. 3a.

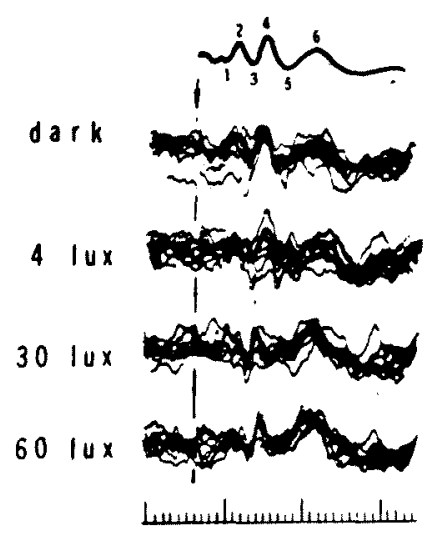

Fig. 3b.

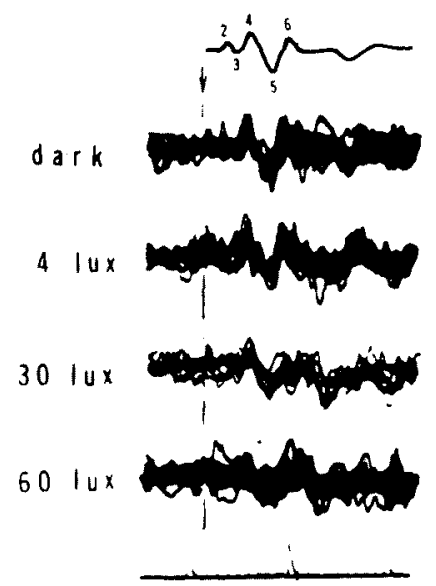

Fig. 3c.

Fig. 3 a, b and c. See text.

dominance from the 4 th to 6 th wave was observed.

Besides the cases mentioned above, there were two cases in which the 4th and 6th waves became fuged to one hump as shown in Fig. 4, and a case in which the amplitude decreased in 4 lux and increased again in 30 and 60 lux into such a shape as seen at the dark level.

Although the evoked potentials change their shapes according to the adaptation level, the latencies and the culmination times of components are scarcely 


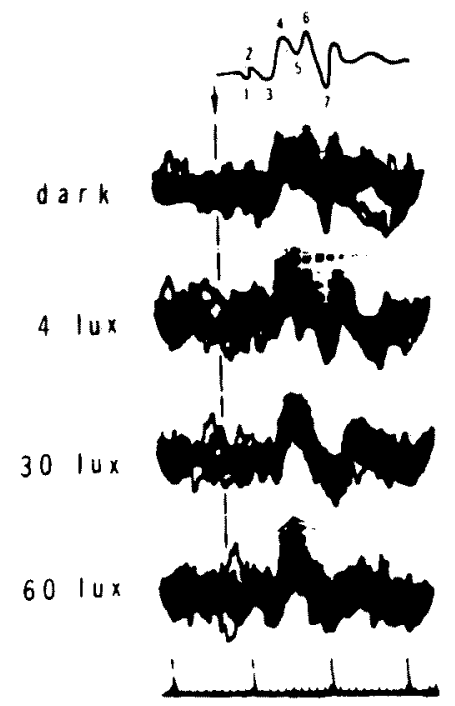

Fig. 4. See text.

delayed under the adaptation from dark to 30 lux, but cases without delay in 60 lux were only exceptionally observed.

2. Changes of evoked potentials due to the reduction of the intensity of stimulation light at various adaptation levels

These experiments were performed in 3 subjects. The intensity of stimulation light was decreased from 0 to $\overline{4}$ in five steps.

In the dark adaptation the changes of the evoked potentials caused by decreasing the intensity of stimulation light were reported in the previous paper. ${ }^{16}$ ) It was not easy to established a rule as to these changes since the change was somewhat different from case to case, but the three general tendencies were observed, i.e. decrease in amplitude, prolongation of latency and culmination time, and shifts of predominant components.

When the adaptation level was changed, these tendencies became less clear. The evoked potentials did not fail to appear within the range of adaptation level from dark to light of $60 \mathrm{lux}$, as mentioned in the preceding chapter when the maximum intensity of stimulation light was used. However, when the intensity of stimulation light was decreased, the evoked potentials became weaker as the adaptation level was raised. For adaptation levels of dark, 4, 30 and 60 lux, the evoked potentials by photic stimuli of intensities $0, \overline{1}, \overline{2}, \overline{3}$, and $\overline{4}$ are shown in Fig. 5. In this figure, the evoked potentials under dark and 4 lux adaptation can be noticed in all intensity ranges of stimulation light. Under the 


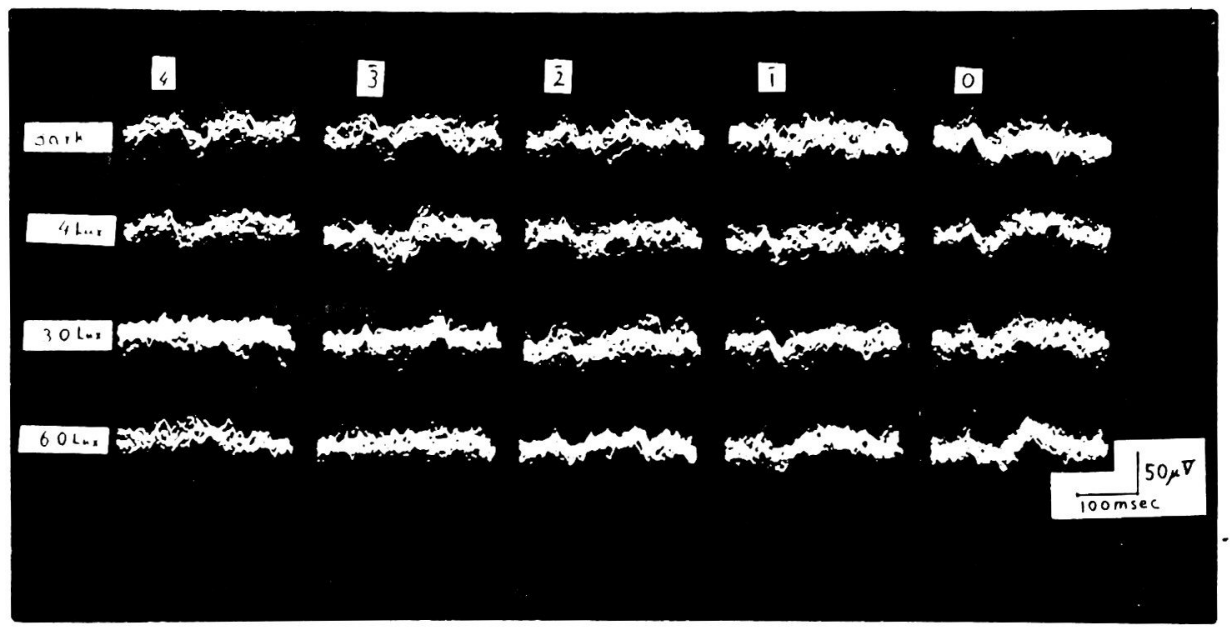
light.

Fig. 5. Evoked potentials at four adaptation levels and five intensities of stimulation

light adaptation of 30 lux the evoked potential was not elicited by the stimulation light of $\overline{4}$, nor under 60 lux adaptation by the intensity of $\overline{3}$ and $\overline{4}$. 'The increase in the intensity of adaptation light tends to inhibit the evoked potentials and increasing the intensity of stimulation light tends to enhance them within the limit of our experiment.

It was reported in the previous paper ${ }^{16)}$ that the latencies and culmination times of some components of the evoked potentials were delayed in accordance with reduction of the intensity of stimulation light under dark adaptation. The latencies and culmination times, with the maximum intensity of stimulation light which we used and at the adaptation level from dark to 60 lux, did not change a great deal, but some exceptional cases showed the prolongation in 60 lux adaptation. When the intensity of the stimulation light was reduced, the changes of the latency and the culmination time could occur under light adaptation. The change of the culmination time of the 2 nd negative wave in one of three cases under 4 adaptation levels and 5 stimulus intensities are illustrated in Fig. 6 . In Fig. 6a, the abscissa indicates the intensity of adaptation light and the ordinate the culmination time. The culmination time of evoked potential by the stimulus of maximum intensity does not change under the adaptation levels from dark to $30 \mathrm{lux}$, but in 60 lux adaptation it shows a slight increase. With stimulating intensity of $\overline{1}$, the culmination time remains unchanged in the range of dark to 30 lux, but the slight shortening of it is observed in 60 lux. For the intensity of $\overline{2}$ the culmination time increases from darkness to 30 lux and the shortening is observed in 60 lux adaptation. For $\overline{\mathbf{3}}$, the culmination time increases from 


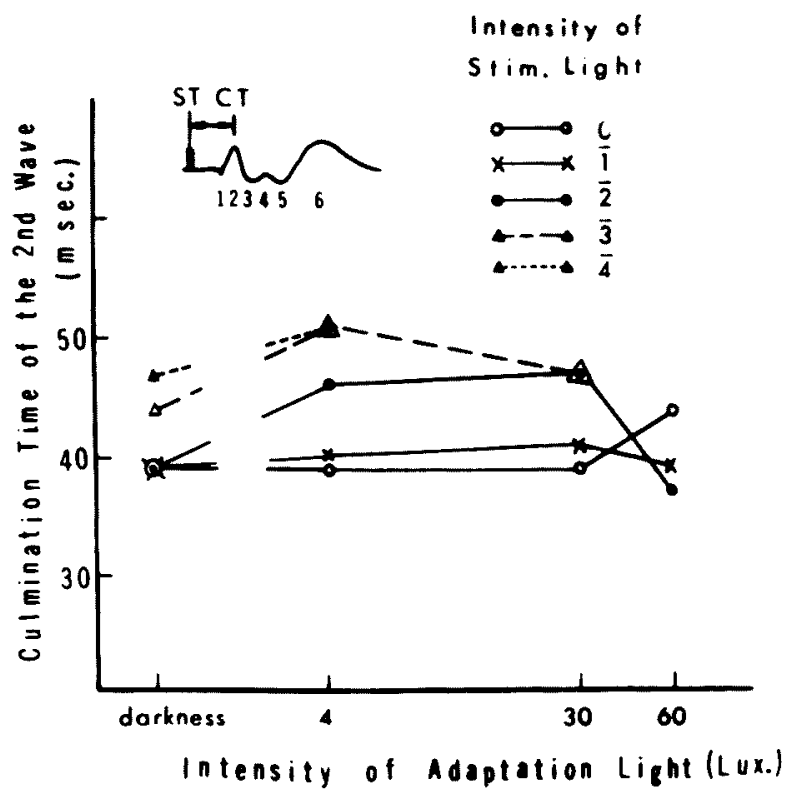

Fig. 6a.

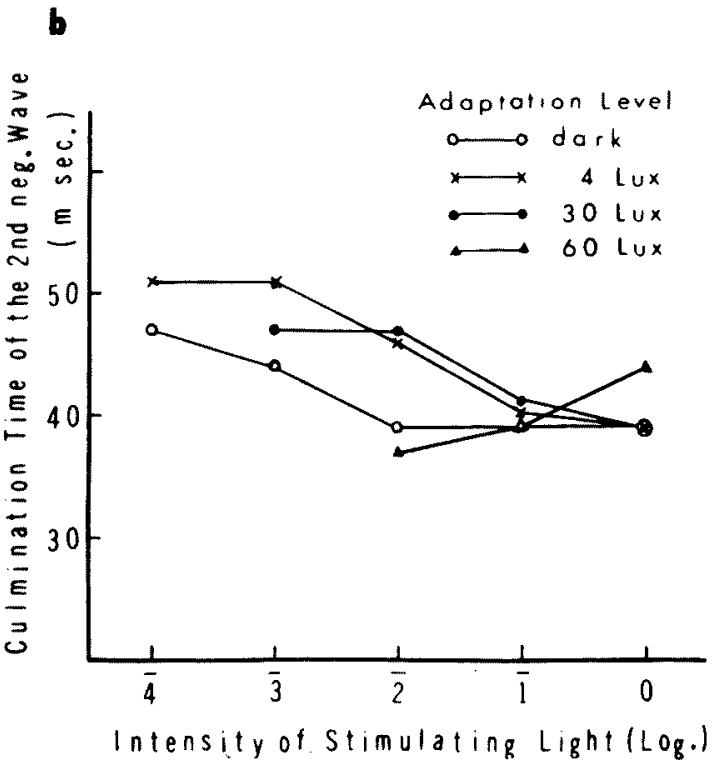

Fig. 6b.

Fig. 6a and b. Culmination time of the 2nd component depending upon intensity of adaptation light (a) and that of stimulation light (b), 
darkness to 4 lux and decreases slightly in $30 \mathrm{lux}$, and in 60 lux the potential disappears. For $\overline{4}$, the potential disappears in 30 lux adaptation. In Fig. 6b culmination times are plotted against the intensity of stimulation light.

\section{DISCUSSION}

It is a well-known fact that the sensitivity of the visual system to light is different according to the condition of adaptation of the retina. It is important to provide information about the effect of the adaptation level upon the evoked potentials in the visual cortex which represents a terminal of the visual system. Some investigators reported on the basis of the results on animal experiments that the initial diphasic wave with the first positive deflection is due to presynaptic neurons in the specific projection system and that the subsequent triphasic wave is due to postsynaptic neurons in the diffuse projection system in the reticular formation. However, it is not always right to say that the subsequent triphasic wave of the human evoked potential is analogous to the one obtained by experiments, since the experimental conditions and the development of the cortical structure are different between man and animal.

It is not easy uniquely to specify the changes of the evoked potentials due to the variation of the adaptation level since the evoked potentials are individually different, but some general tendencies can be seen in them, for example the decreasing of amplitude and the fluctuation of predominance of some components.

When the stimulus of maximum intensity was used, only little effect of adaptation could be observed, as has been shown above, but when the intensity of the stimulus was reduced the evoked potentials disappeared upon increasing the intensity of the adaptation light. The elicitation of the evoked potentials depends upon the intensity of the stimulation light against the intensity of adaptation light, and it seems that the same relation applies to our visual sensation; we percept in darkness such a faint light as hardly perceptible in light. In the dark-adapted state, the culmination time of the evoked potential is always delayed by reduction of the intensity of stimulation light, but in light adaptation either prolongation or shortening of culmination time is observed according to the intensity of adaptation light relative to that of stimulation light.

In our experiments no mydriatic was applied to the subject's eyes, so that the energy of stimulation light falling upon the retina was different according to the level of adaptation. When the adaptation light changes from $10^{-6}$ to $10^{4}$, the pupil changes from the maximum diameter of $8 \mathrm{~mm}$ to the minimum of $2 \mathrm{~mm}$ according to Reeves. ${ }^{19}$ ) Hence, the possible maximum reduction of energy is only one-sixteenth. Therefore, it seems that the reduction of energy due to pupilary contraction would be negligible, in view of the narrow range of intensities of adaptation light in our experiments. 
The physiological mechanism of these changes of the evoked potentials against the change of the level of adaptation is to be clarified in further experiments.

\section{CONCLUSION}

The evoked potentials of the visual cortex by a flash of light were analyzed in 37 subjects under the conditions of dark adaptation and light adaptation to 4,30 and 60 lux. Although some individual differences were observed concerning the effect of light adaptation upon the evoked potentials two tendencies were observed, i.e. the decrease of amplitude and changes in relative dominance among the components of evoked potential. The culmination times of some components of exoked potential were prolonged in dark adaptation when the intensity of the stimulus was reduced Either prolongation or shortening was caused by the reduction of the intensity of stimulation depending upon the adaptation level.

Authers express their thanks to Prof. K. Motokawa, Tohoku University, for his advice and criticism.

\section{References}

1) Monnier, M. \& Berger, G.P., Rev. Neurol., 1952, 87, 189.

2) Monnier, M. \& Berger, G.P., Opthalmologica, 1953, 129, 15.

3) Monnier, M. \& Dieterle, P., Bull. Acad. Suisse Sci. Méd., 1954, 10, 113.

4) Cobb, W. \& Morton, H.B., J. EEG Clin. Neurophysiol., 1952, 4, 547.

5) Cobb, W. \& Dawson, G.D., J. Physiol, 1960, 152, 108.

6) Ciganék, L., Rev. Neurol,, 1958, 99, 194.

7) Ciganék, L., J. EEG Clin. Neurophysiol., 1959, 11, 65.

8) Ciganék, L., ibid 1961, 13, 165.

9) Balen, A.T.M. van \& Henkes, H.E., ibid 1960, 44, 449.

10) Balen, A.T.M. van \& Henkes, H.E., Brit. J. Opthal., 1962, 46, 12.

11) Laue, H. \& Monnier, M.. Pflügers Archiv ges. Physiol., 1954, 259, 231.

12) Laue, H., v. Graefes Arch. Ophthal., 1961, 163, 337.

13) Calvet, J., Cathala, H.P., Hirsch, J.F. \& Scherrer, J., C.R. Soc. Biol. Paris, 1956, 150, 1348

14) Contamin, F. \& Cathala, H.P., J. EEG Clin. Neurophysiol., 1961, 13, 674.

15) Hirsch, J.F., Pertuiset, B., Calvet, J., Buisson-Ferey, J., Fischgold, H. \& Scherrer, J., J. EEG. Clin. Neurophysiol., 1961, 13, 411

16) Ebe, M. \& Mikami, T., Tohoku J. Exp. Med., 1962, 78, 17

17) Dawson, G.D., J. Neurol. Neurosurg. Psychiat., 1947, 10, 134

18) Ebe, M., Mikami, T., Aki, \& Miyazaki, M., Tohoku J. Exp. Med., 1962, 77, 352

19) Reeves, cited from Satake, Y. \& Fujita, T., Seirigaku Kogi (Jap.), 1950, Nanzando. Tokyo. 